13th ICATPP Conference on

Astroparticle, Particle, Space Physics and Detectors for Physics Applications

Como, Italy, October 2011

\title{
Validation of the Local Hadronic Calibration Scheme of ATLAS with Combined Beam Test Data in the End-Cap and Forward Regions of ATLAS
}

\author{
A. Kiryunin and P. Strizenec \\ (on behalf of the ATLAS Liquid Argon End-Cap Collaboration)
}

Parallel session VII

on Calorimetry and Applications II

October 4, 2011 


\section{Outline}

- Introduction

- Topological clustering algorithm

- Local hadronic calibration scheme

- Beam tests of the combined setup of ATLAS end-cap and forward calorimeters

- Monte Carlo simulations of the combined beam tests

- Results of the validation of the local hadronic calibration scheme with beam test data

- Conclusions 


\section{Introduction}

\section{Calorimeter system of the ATLAS detector}

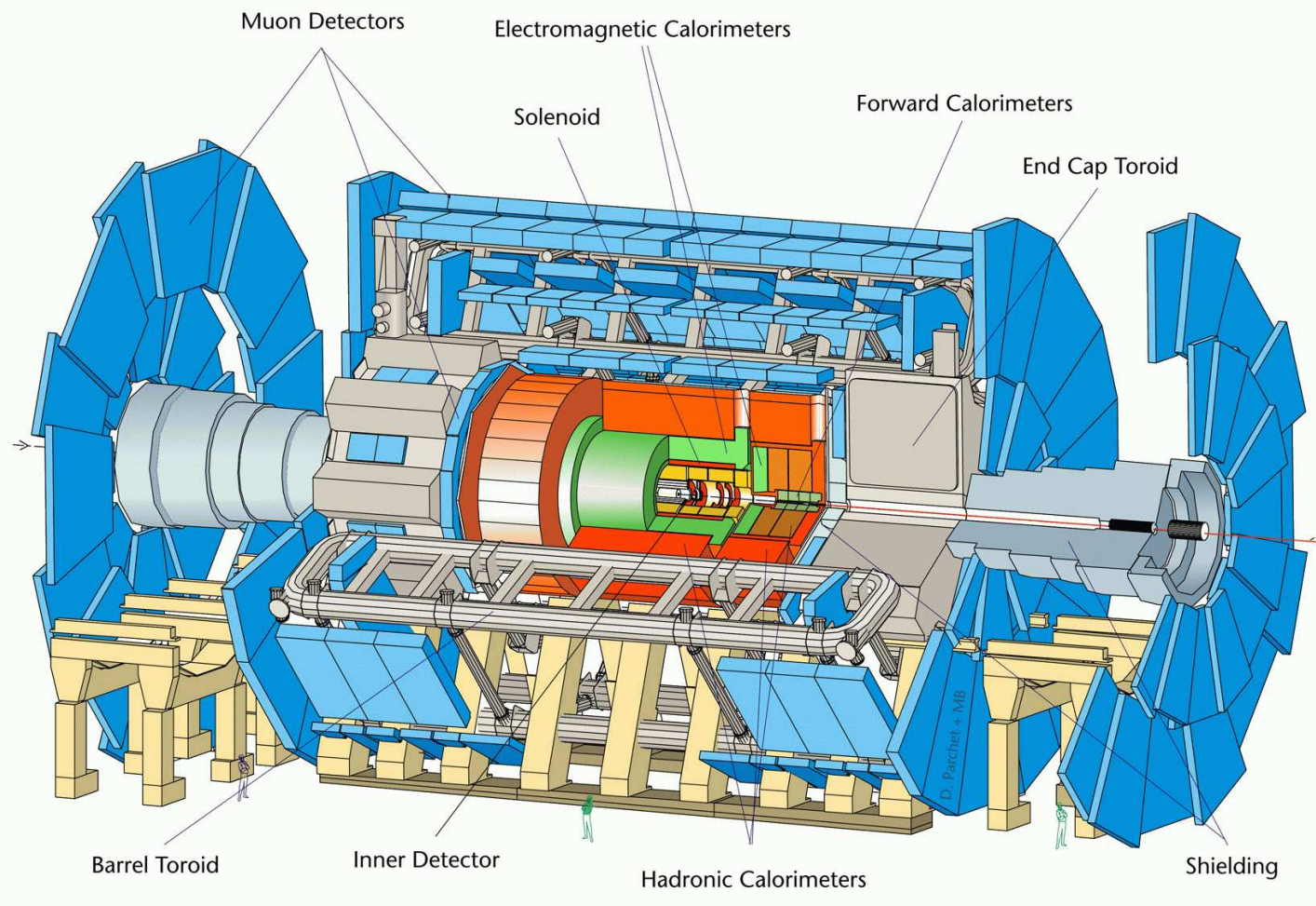

- Electromagnetic calorimeters: lead/liquid argon (LAr)

- barrel: $|\eta|<1.475$

- end-cap: $1.375<|\eta|<3.2$

- Hadronic Tile calorimeter: iron/scintillator

- barrel: $|\eta|<1.0$

- extended barrel: $0.8<|\eta|<1.7$

- Hadronic end-cap calorimeter: copper/LAr, $1.5<|\eta|<3.2$

- Forward calorimeter: $3.1<|\eta|<4.9$

- electromagnetic module: copper/LAr

- hadronic modules: tungsten/LAr

$\begin{array}{ccccc}\text { Coverage } & \text { Number of channels } & \text { Weight } & \text { Length } & \text { Diameter } \\ |\eta|<4.9 & \sim 200000 & 4000 \text { tons } & \sim 13 \mathrm{~m} & \sim 8.5 \mathrm{~m}\end{array}$




\section{Energy reconstruction and calibration in ATLAS calorimeters}

1. Measurement of energy at electromagnetic (EM) scale in all calorimeter cells

2. Reconstruction of clusters from calorimeter cells

3. Application of the hadronic calibration scheme to correct for:

- invisible energy due to the non-compensating nature of ATLAS calorimeters

- energy losses in regions not instrumented with read-out

- energy losses due to unavoidable (at LHC conditions) threshold cuts in the signal reconstruction

4. Jet finding, missing transverse energy reconstruction with calibrated clusters as an input

5. Further energy corrections (to restore the true jet energy, for example)

\section{Local hadronic calibration ( LC)}

- LC is one of the hadronic calibration schemes developed for the ATLAS calorimeter system

- Input for the local hadronic calibration is topological clusters reconstructed at EM-scale

- Validation of this calibration scheme is done with data obtained during combined beam tests of modules of ATLAS end-cap and forward calorimeters 


\section{D Topological Clusters \\ Clustering algorithm}

- Cells with absolute energy $|E|>4 \sigma_{\text {noise }}$ seed clusters

- All neighbouring cells (within the calorimeter layer and those overlapping in $\eta$ and $\varphi$ in adjacent layers and/or calorimeter systems) are added to the cluster

- Cells with $|E|>2 \sigma_{\text {noise }}$ also collect all their neighbours within the cluster

- Two clusters are merged if they share a cell with absolute energy above $2 \sigma_{\text {noise }}$

- Clusters are split around local cell maxima with a fixed threshold of $E=500 \mathrm{MeV}$

- Two clusters including the same cell share its energy

\section{Cluster moments}

- Cluster moments describe shapes and characteristics of reconstructed clusters

$$
<x^{n}>=\sum_{i} E_{i} x_{i}^{n} / \sum_{i} E_{i}
$$

where $i$ runs over all cluster cells with $E>0$

- Moments used for the local hadronic calibration:

- cell energy density $\rho$

- shower depth $\lambda_{\text {center }}$ - distance between a shower center and a calorimeter front face

- isolation parameter - fraction of cells neighbouring the cluster not included in other clusters 


\section{Local hadronic calibration scheme}

1. Classification of clusters as electromagnetic or hadronic

2. Weighting of cluster energy according to the cluster origin

3. Out-of-cluster corrections: to correct for energy deposits inside the calorimeter but outside calorimeter clusters due to the noise thresholds

4. Dead material corrections: to compensate for energy deposits in materials outside of active calorimeter volumes

- Steps 2-4 may be applied in any order

- Weights and corrections are determined from the detailed Monte Carlo (MC) simulations of single pions

- LC is based fully and solely on calorimetric signal features

- It does not depend on initial physics processes 


\section{Combined beam tests of ATLAS end-cap and forward calorimeters}

- Beam tests in 2004 in the H6 beamline at the CERN SPS

- Modules of three ATLAS calorimeters:

- electromagnetic end-cap EMEC

- hadronic end-cap HEC

- forward calorimeter FCal (FCal1 - electromagnetic module, $\mathrm{FCal} 2$ - hadronic module)
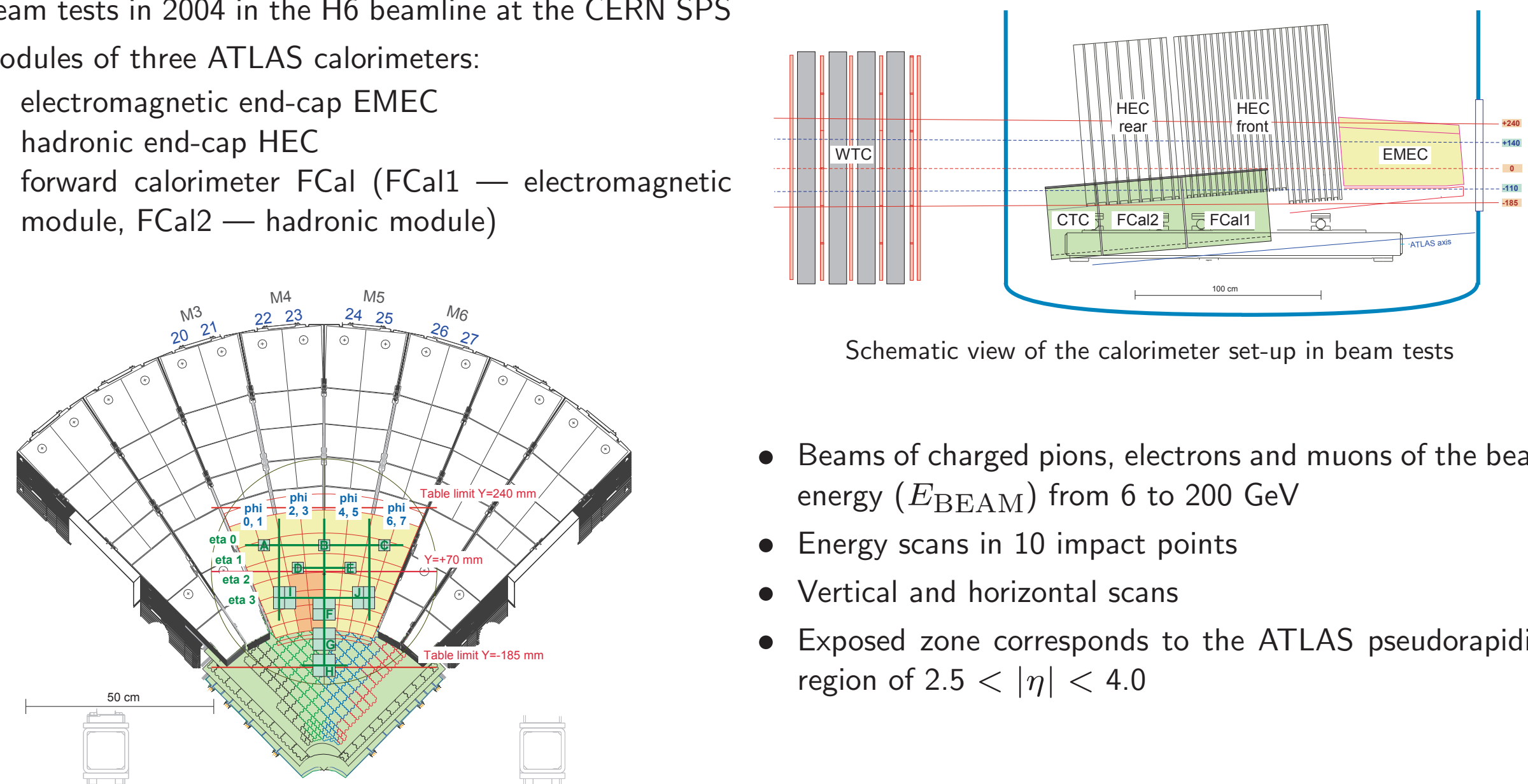

Schematic view of the calorimeter set-up in beam tests

- Beams of charged pions, electrons and muons of the beam energy ( $\left.E_{\mathrm{BEAM}}\right)$ from 6 to $200 \mathrm{GeV}$

- Energy scans in 10 impact points

- Vertical and horizontal scans

- Exposed zone corresponds to the ATLAS pseudorapidity region of $2.5<|\eta|<4.0$

Position of scanned points on the front face of the calorimeter 


\section{Simulations}

- GEANT4 simulation toolkit (version 9.2)

- Used physics lists (set of processes for the simulation of hadronic interactions):

- QGSP-BERT (Quark-Gluon-String and Bertini cascade models)

- FTFP-BERT (FRITIOF string model and Bertini cascade model)

- Two set of MC samples were produced:

1. Simulation of charged and neutral pions - to derive weights and correction coefficients for the local hadronic calibration

- flat coverage of the whole beam test space: $\pi / 2<\varphi<\pi$ and $2.0<\eta<4.0$

- beam energies in the interval from 1 to $400 \mathrm{GeV}$ logarithmically flat

- storing "calibration hits" (energy depositions due to ionization, invisible and escaped energies in active and passive parts of the beam test setup)

2. Simulations of energy and position scans with charged pions and electrons - to compare with available experimental data 


\section{Results of the LC validation with test beam data}

- Energy scans in the end-cap region ( $\eta \simeq 2.75$ in ATLAS)

- electrons

- charged pions

- Energy scans in the forward region $(\eta \simeq 3.6$ in ATLAS)

- electrons

- charged pions

- Vertical (i.e. pseudorapidity) scan with charged pions over the entire calorimeter system

\section{Energy scans}

- Energy reconstruction:

- sum of energies of all clusters

- Gaussian fit within $\pm 3 \sigma$ around peak value $E_{0}$

- Analysed variables:

- cluster moments: energy density and shower depth

- pion energy response $E_{0} / E_{\mathrm{BEAM}}$

- pion energy resolution $\sigma / E_{0}$ 


\section{Electrons in the end-cap region}

- Six beam energies: from 6 to $193 \mathrm{GeV}$

- MC simulations with QGSP-BERT

- Average number of clusters per beam electron: 1.2 (for data and MC)

Average energies in the EMEC
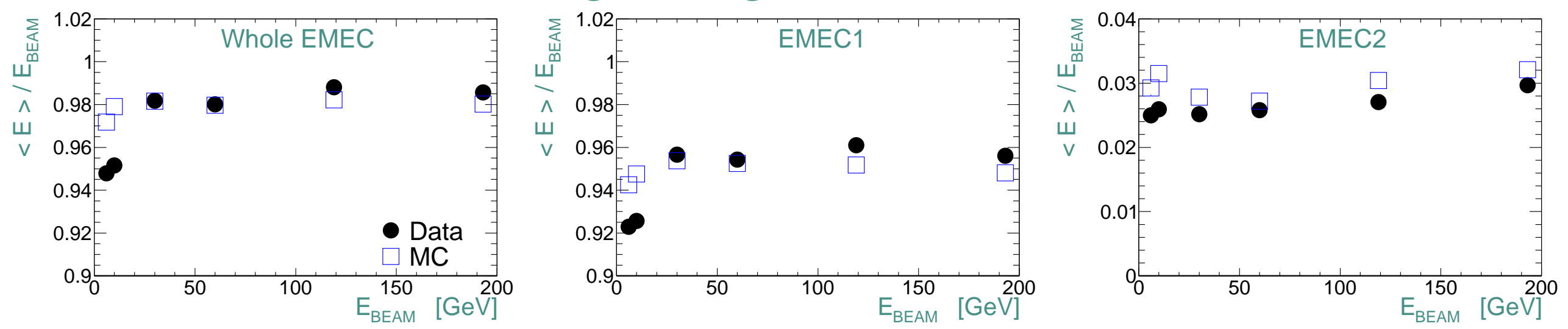

- Good agreement between data and $\mathrm{MC}$ predictions (for $E_{\mathrm{BEAM}} \geq 30 \mathrm{GeV}$ )

- Few percent difference between the measured energies and the corresponding beam energies:

- energy losses in the material in front of the EMEC

- longitudinal leakage to the HEC 
Electrons in the end-cap region

Cluster moments
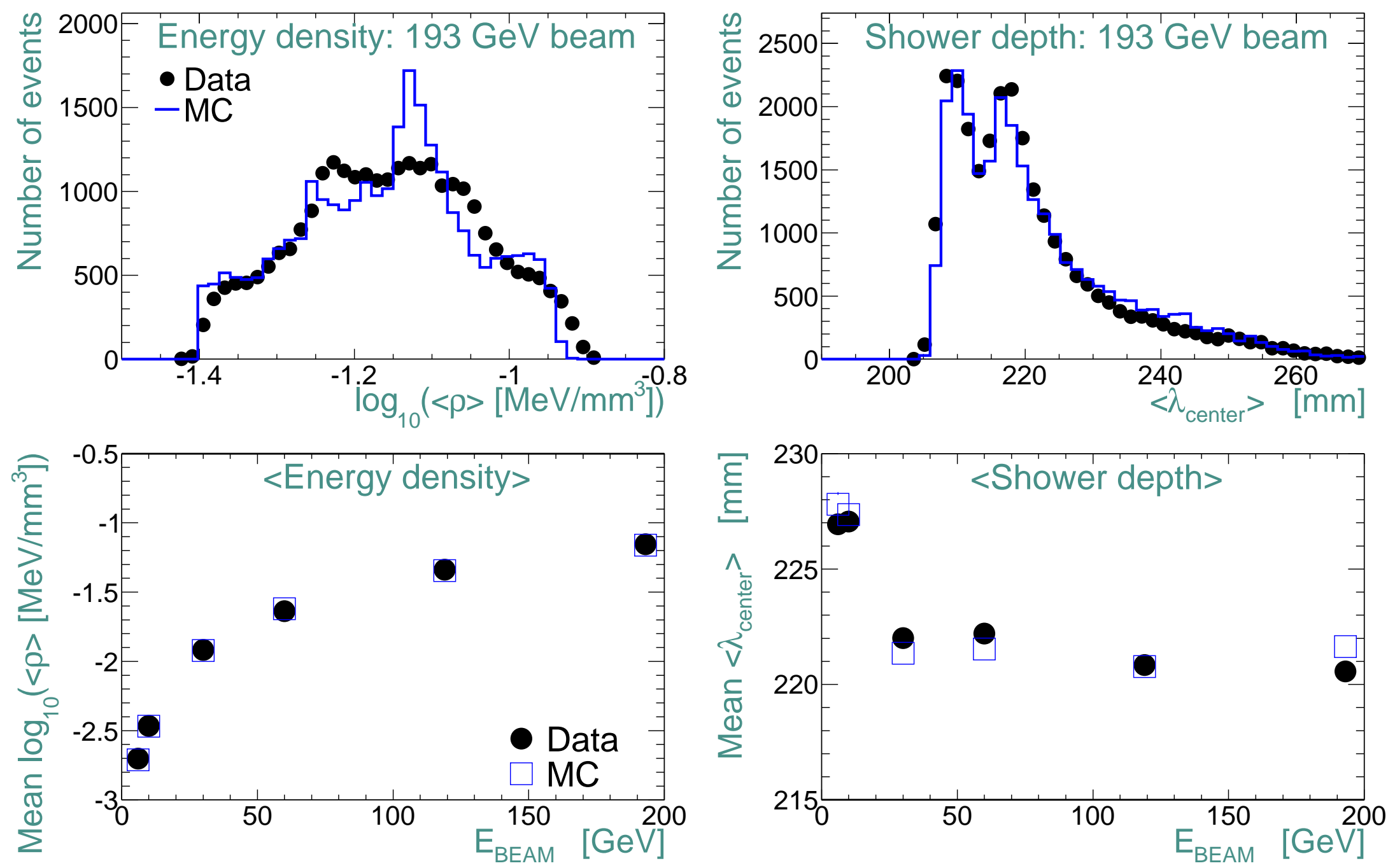

Reasonably good agreement between data and MC 


\section{Charged pions in the end-cap region}

- Seven beam energies: from 10 to $200 \mathrm{GeV}$

- MC simulations with the two physics lists: QGSP-BERT and FTFP-BERT

- Average number of clusters per beam pion:

\begin{tabular}{lcc}
\hline$E_{\text {BEAM }}$ & $10 \mathrm{GeV}$ & $200 \mathrm{GeV}$ \\
\hline Data & 1.6 & 3.0 \\
Both MC & 1.6 & 2.6 \\
\hline
\end{tabular}

Cluster moments
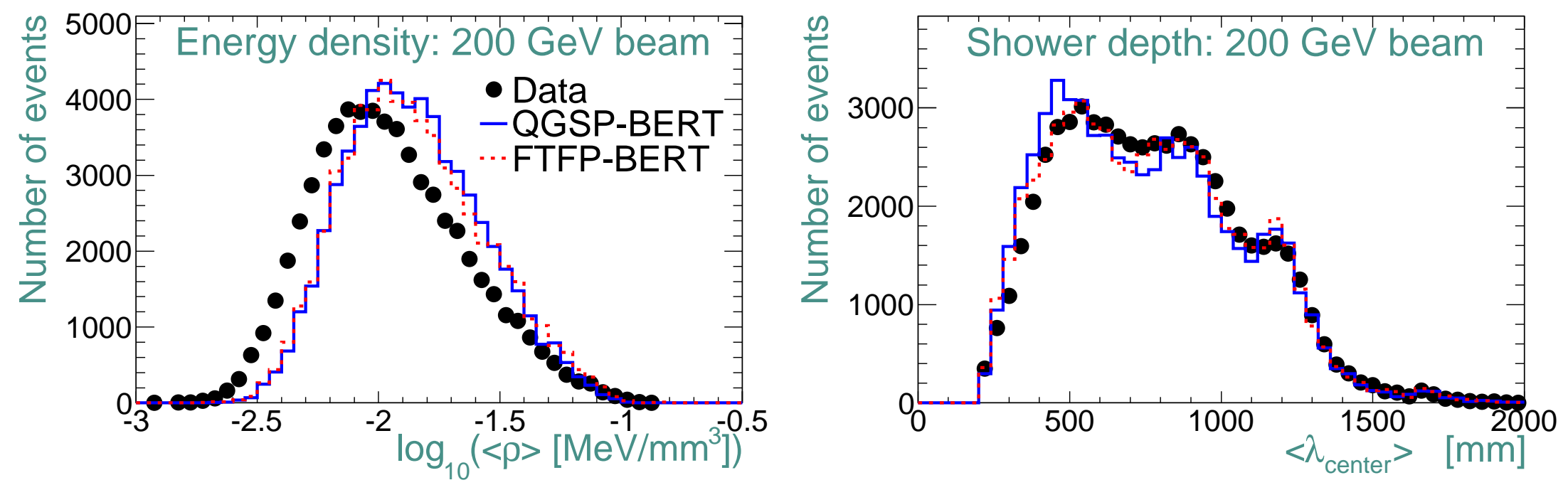
Cluster moments (cont'd)
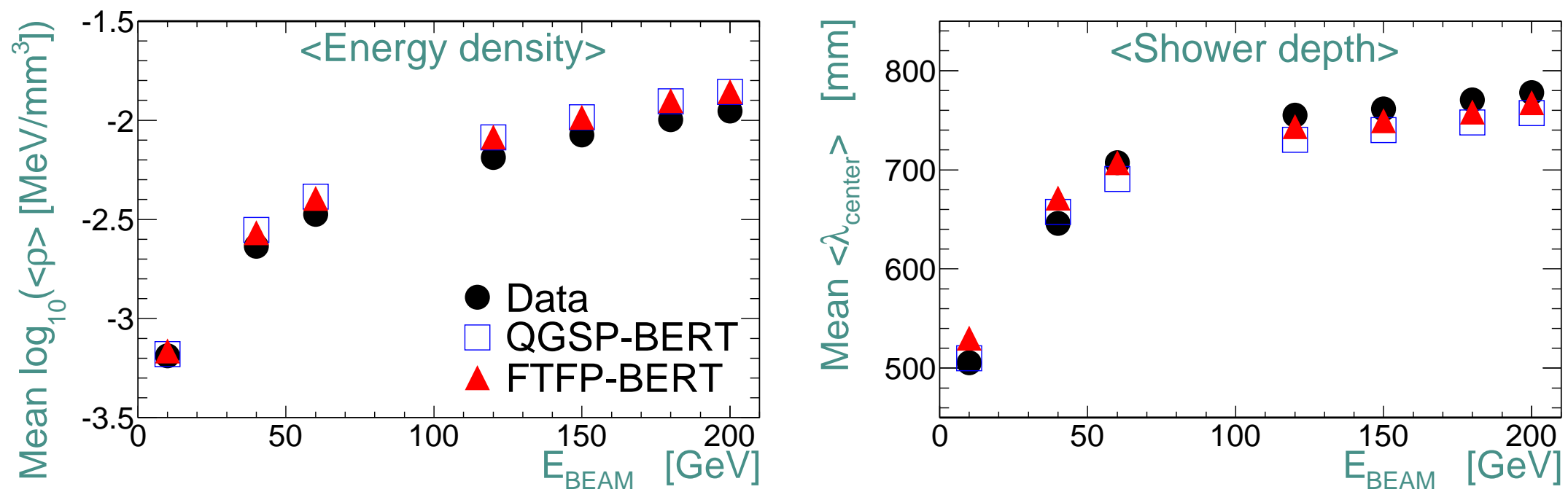

- Average energy density predicted by both simulations is 3-6 \% larger than in the data (for $E_{\mathrm{BEAM}} \geq$ $40 \mathrm{GeV}$ ). Hadronic showers are hence more compact in simulations than in data.

- Difference between MC predictions and data for the mean values of shower depth stays below $3 \%$ (for $E_{\mathrm{BEAM}} \geq 60 \mathrm{GeV}$ ).

- In general, there is agreement between the two simulations and the data in the end-cap region for all studied cluster moments except for those which are related to the transverse shape of hadronic showers. For them the MC predicts a smaller shower size. 


\section{Energy response}

- Ratio of the response (MC to Data): $R=\frac{\left(E_{0} / E_{\mathrm{BEAM}}\right)_{\mathrm{MC}}}{\left(E_{0} / E_{\mathrm{BEAM}}\right)_{\text {Data }}}=\frac{E_{0}^{\mathrm{MC}}}{E_{0}^{\text {Data }}}$

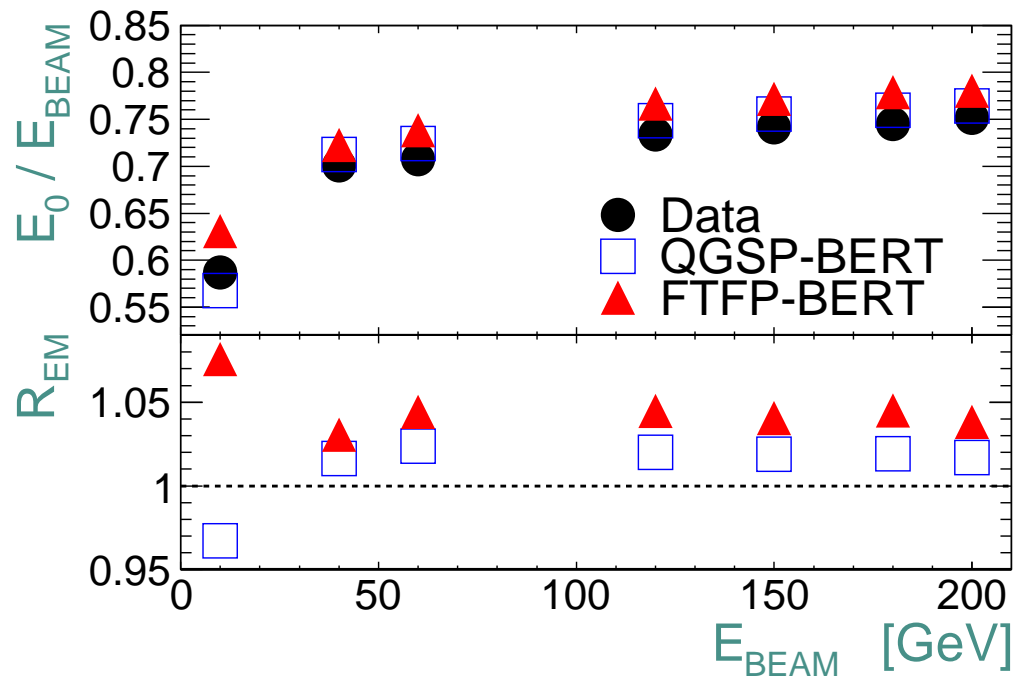

Successive application of LC-steps

$+\mathrm{W} \quad$ cluster classification and weighting

$+\mathrm{OOC}$ out-of-cluster corrections

+ DM dead material corrections $4 \%$

\section{Response at EM-scale}

- Non-linear response indicates the non-compensating nature of end-cap calorimeters

- QGSP-BERT overestimates the experimental response by $2 \%$, while FTFP-BERT overestimates the data by

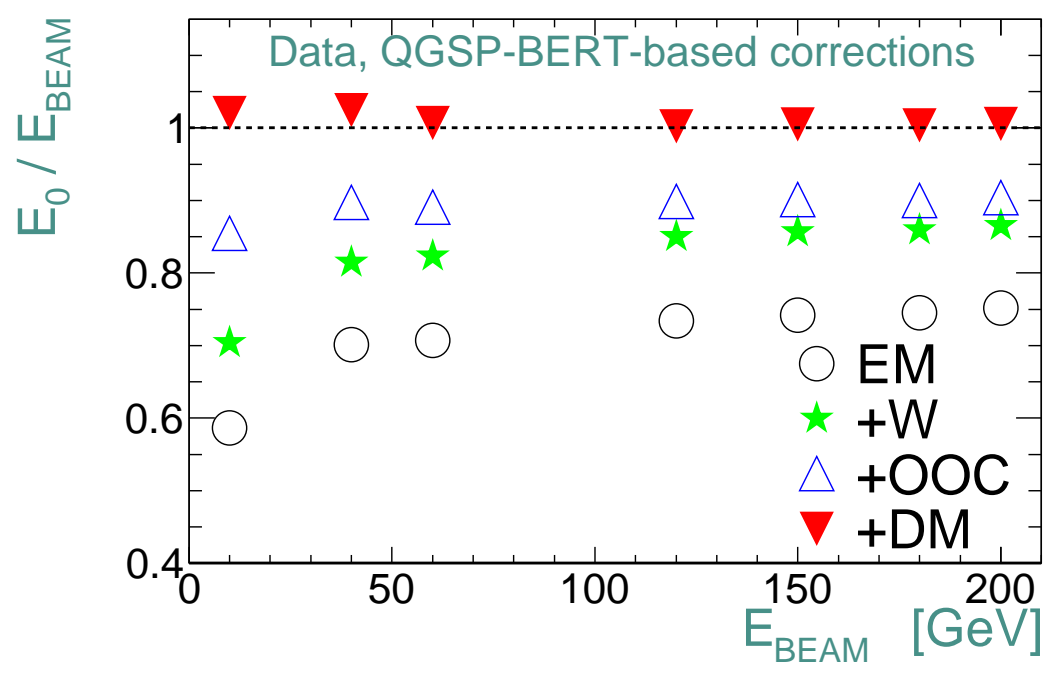


Charged pions in the end-cap region

\section{Energy response at LC-scale}
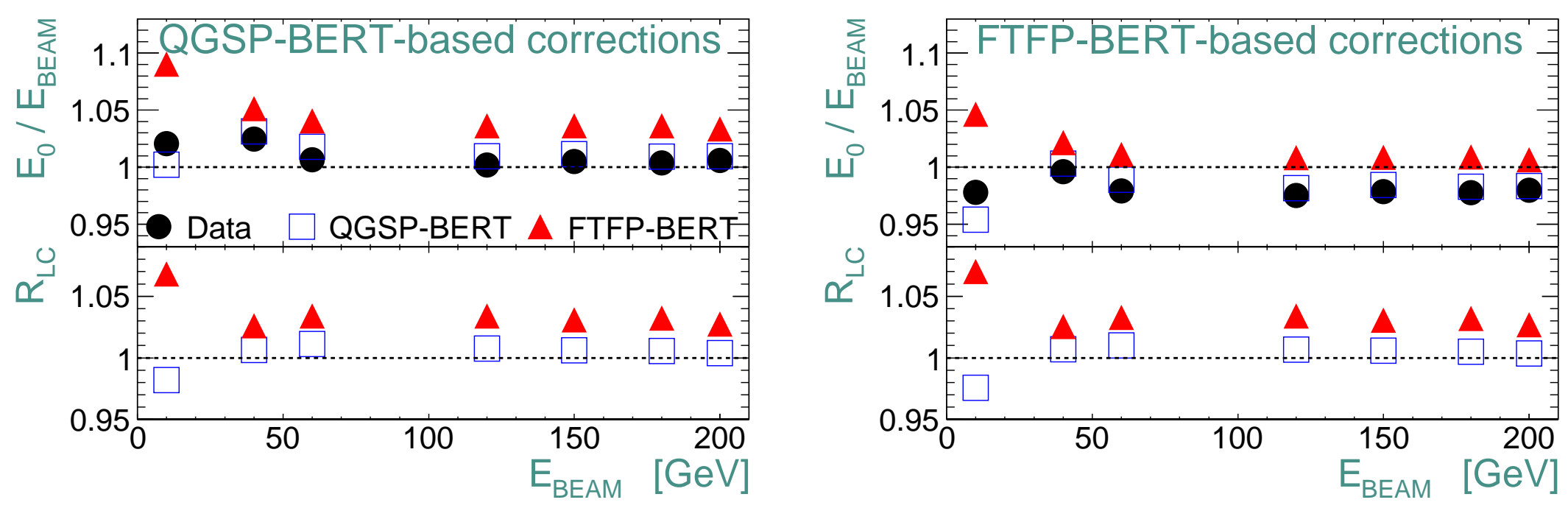

- The LC-scale experimental response is correctly reconstructed by the QGSP-BERT-based corrections

- The application of the native LC-weights and corrections to the corresponding simulations slightly overestimates the energy response

- Agreement between data and MC predictions is better at LC-scale than at EM-scale 
Charged pions in the end-cap region

\section{Energy response (cont'd)}

\section{Difference in the response for QGSP-BERT and FTFP-BERT physics lists}

- $\sim 2 \%$ at EM-scale

- $\sim 0 \%$ after application of the native LC-weights and corrections

- $\sim 5 \%$ after application of the non-native LC-weights and corrections

\section{Double ratio of the response}

- To assess the systematic uncertainty of the local hadronic calibration, the EM-scale difference should be factored out

- Double ratio of the response $=R_{\mathrm{LC}} / R_{\mathrm{EM}}$
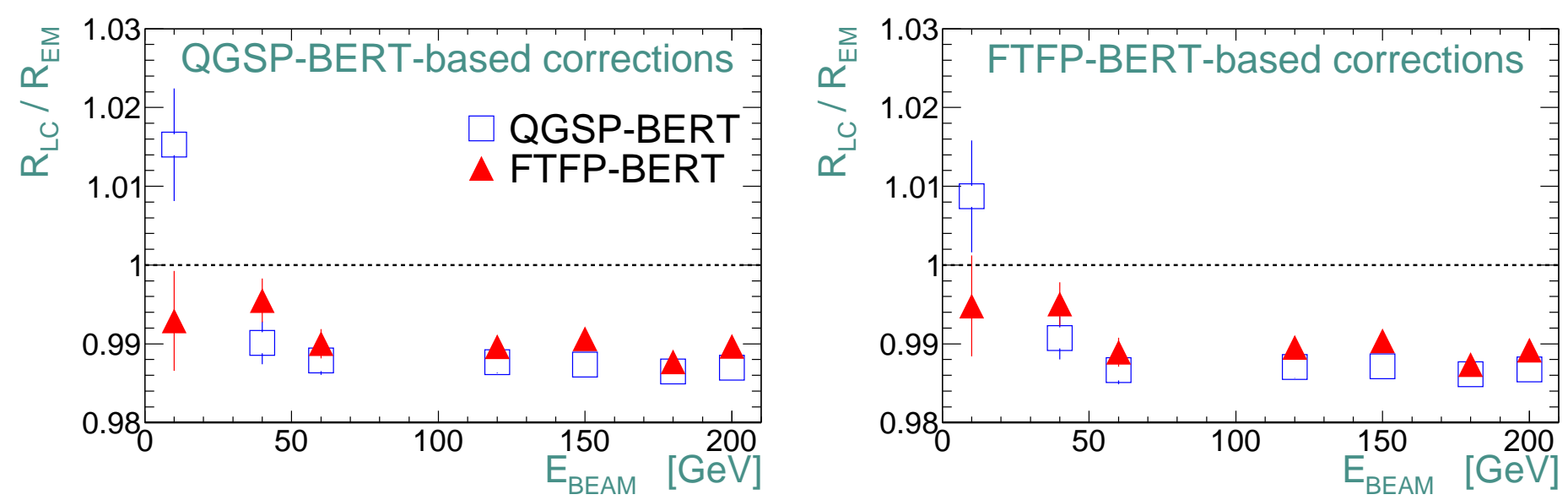

- Double ratio of the energy response differs from unity by 1.0-1.3\%

- Similar results are obtained for both physics lists 
Charged pions in the end-cap region

\section{Energy resolution}

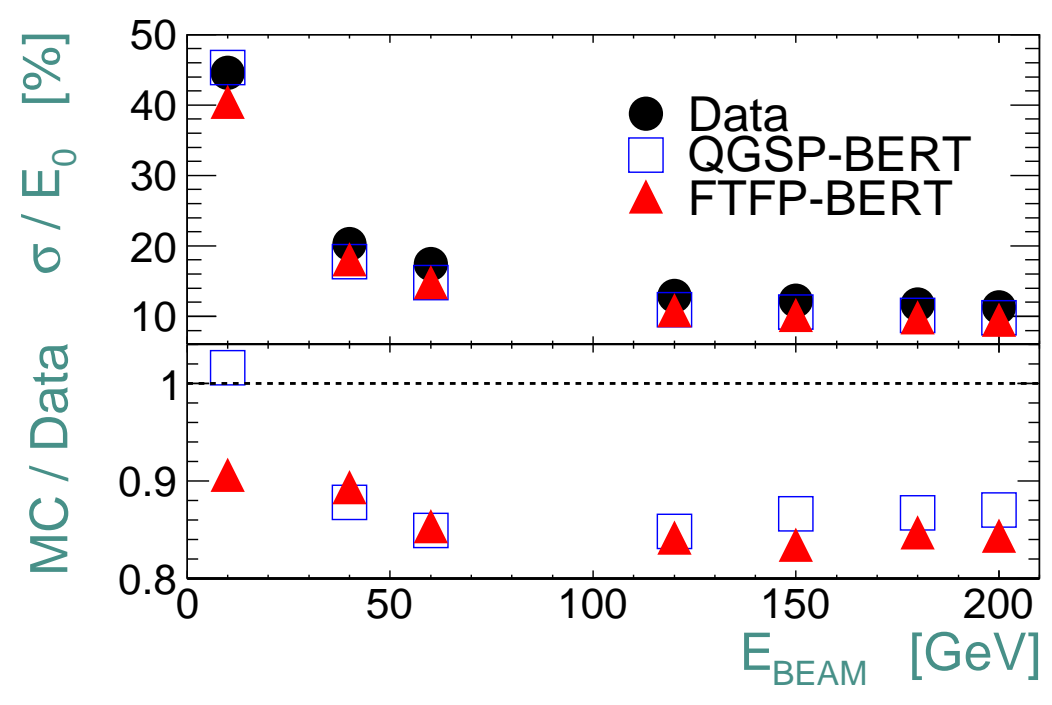

\section{Successive application of LC-steps}

- Weighting step improves the energy resolution at higher beam energies

- Out-of-cluster corrections improve the energy resolution at lower beam energies

\section{Resolution at EM-scale}

- Two physics lists give close results (for $E_{\mathrm{BEAM}} \geq$ $40 \mathrm{GeV}$ )

- They predict better resolution than experimental values by $10-15 \%$

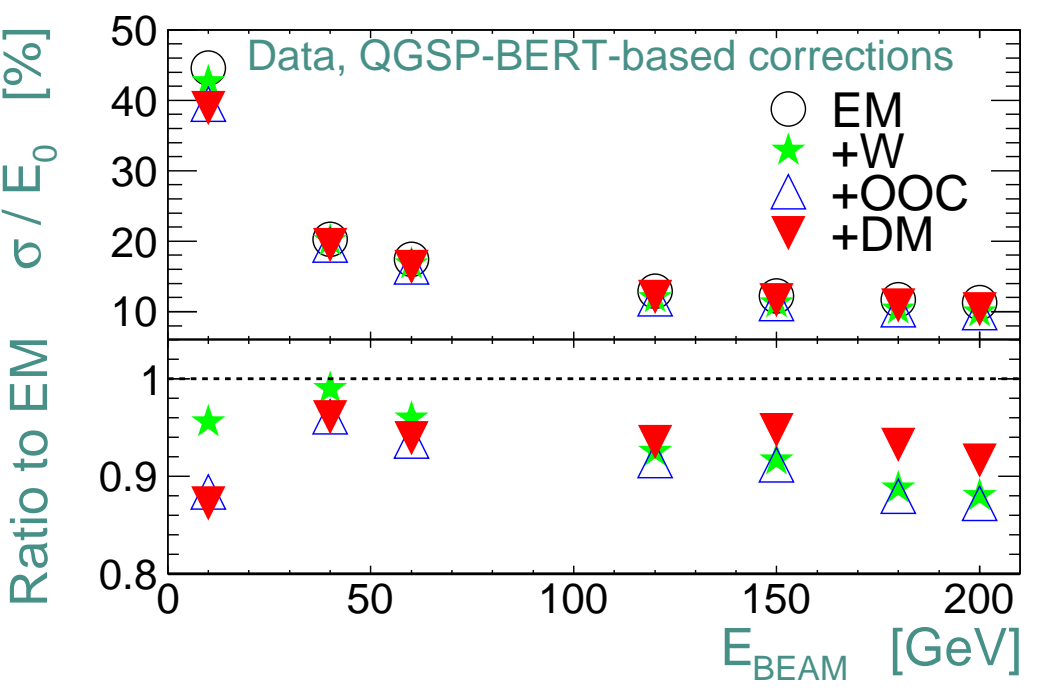


Charged pions in the end-cap region

\section{Energy resolution at LC-scale}
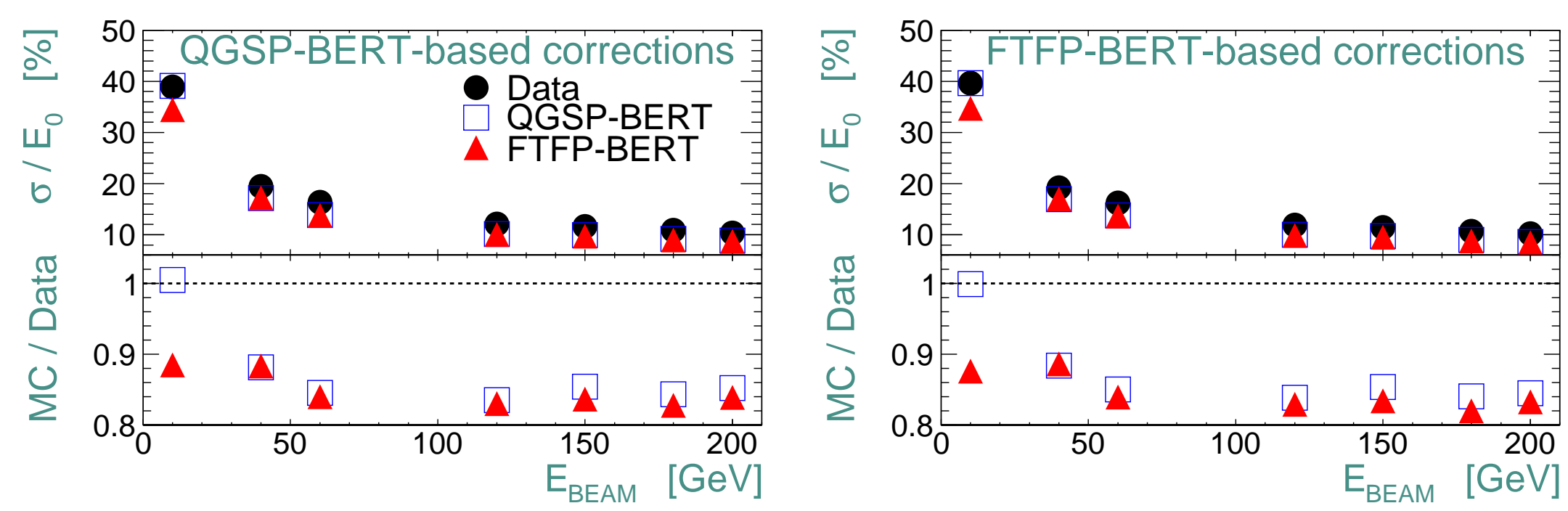

- Improvement of the energy resolution after application of the local hadronic calibration is about $5-15 \%$ for data and simulations

- Resolution improvement at LC-scale with respect to EM-scale is slightly better for simulations than for experimental data 


\section{Electrons in the forward region}

- Six beam energies: from 6 to $193 \mathrm{GeV}$

- MC simulations with QGSP-BERT

- Average number of clusters per beam electron:

varies from 1.3 at $6 \mathrm{GeV}$ to 1.9 at $193 \mathrm{GeV}$ (for data and MC)

Average energies in the FCal1 (electromagnetic module)

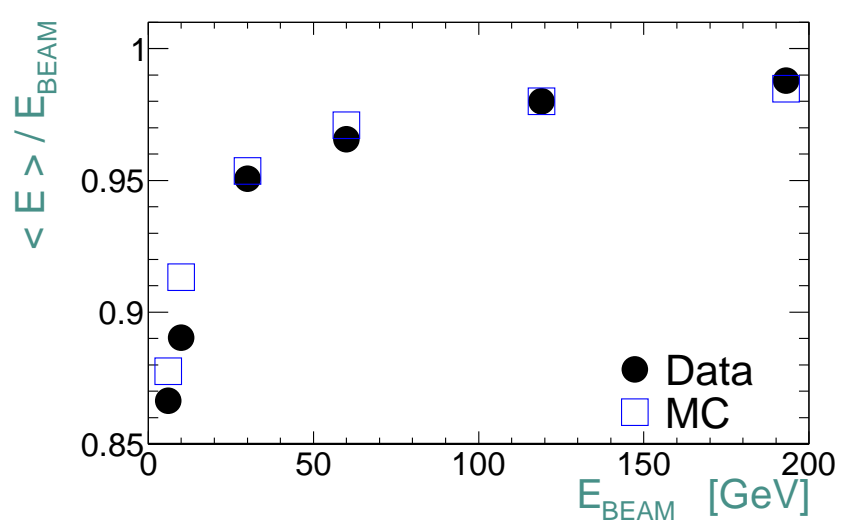

- Good agreement between data and MC predictions (for $E_{\mathrm{BEAM}} \geq 30 \mathrm{GeV}$ )

- Difference between the measured energies and the corresponding beam energies is due to energy losses in the material in front of the FCal 
Electrons in the forward region

\section{Cluster moments}
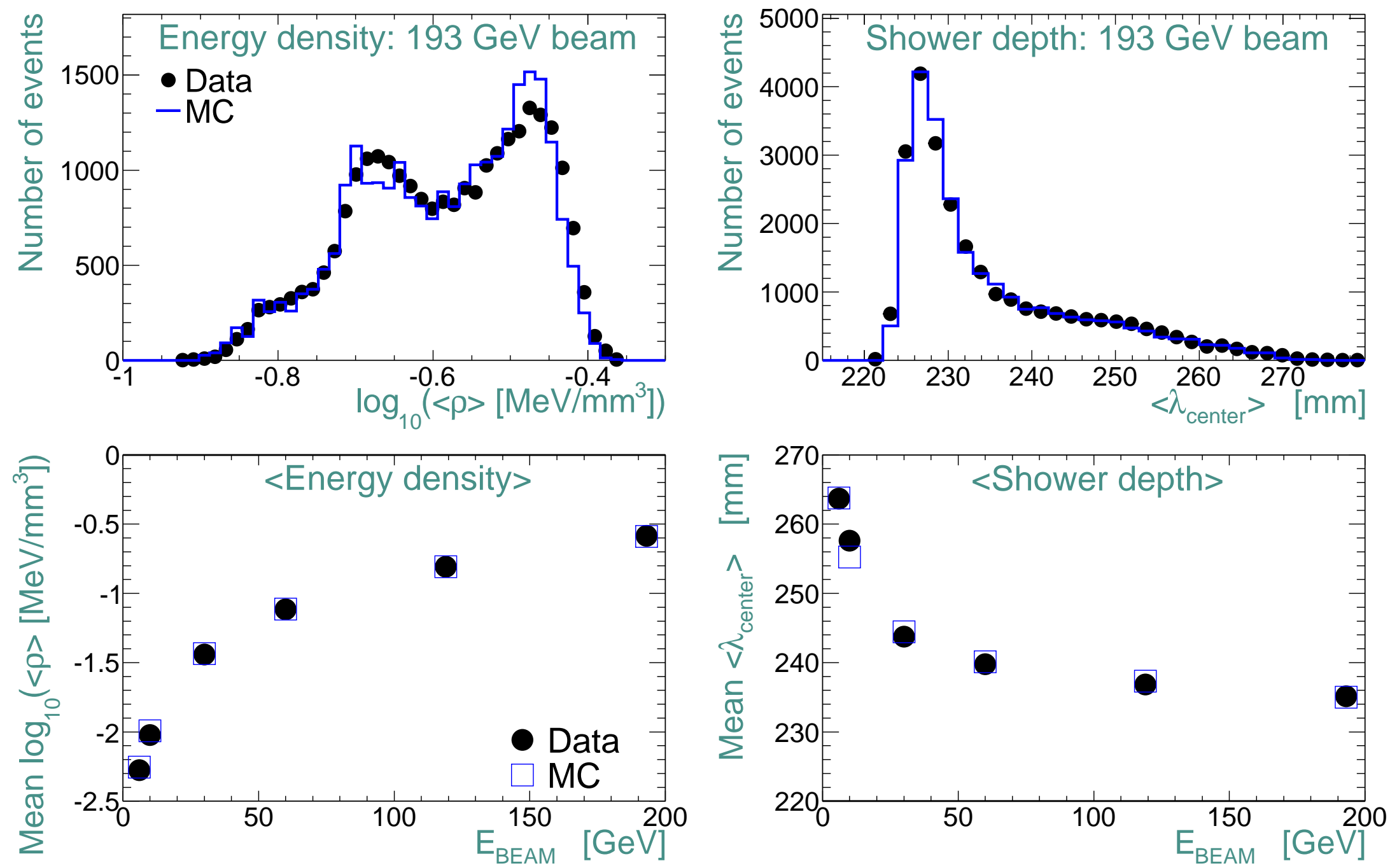

Good agreement (within $1 \%$ ) between data and MC 


\section{Charged pions in the forward region}

- Seven beam energies: from 10 to $200 \mathrm{GeV}$

- MC simulations with the two physics lists: QGSP-BERT and FTFP-BERT

- Average number of clusters per beam pion:

\begin{tabular}{lcc}
\hline$E_{\text {BEAM }}$ & $10 \mathrm{GeV}$ & $200 \mathrm{GeV}$ \\
\hline Data & 1.6 & 3.4 \\
QGSP-BERT & 1.5 & 3.2 \\
FTFP-BERT & 1.6 & 3.1 \\
\hline
\end{tabular}

\section{Cluster moments}
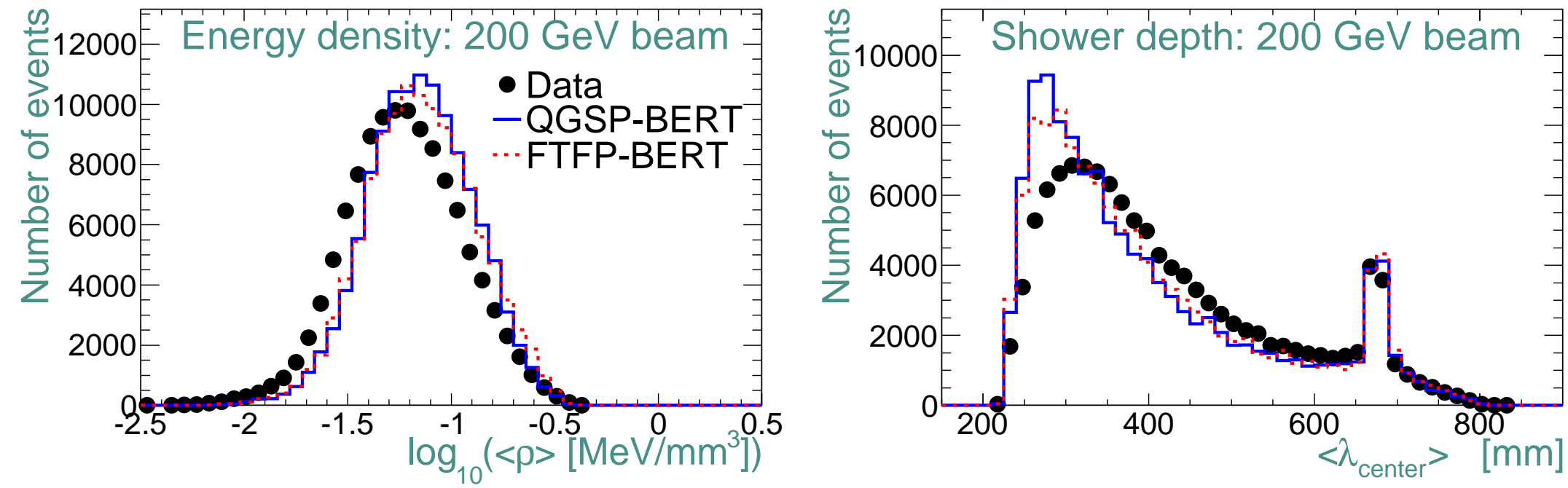
Charged pions in the forward region

Cluster moments (cont'd)
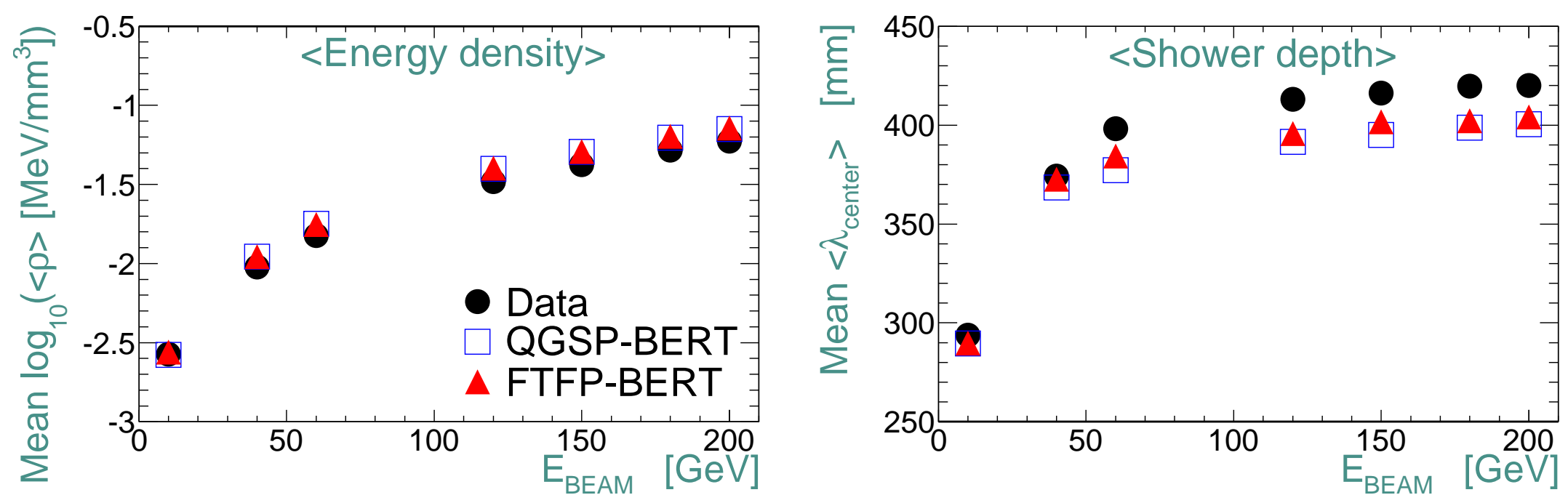

- Both physics lists predict similar shapes of hadronic showers

- Showers are more dense and have smaller depth in the simulations than in the data

- In general, MC predicts earlier start of hadronic showers and they are more compact than observed experimentally in the forward region 
Charged pions in the forward region

\section{Energy response at EM-scale}

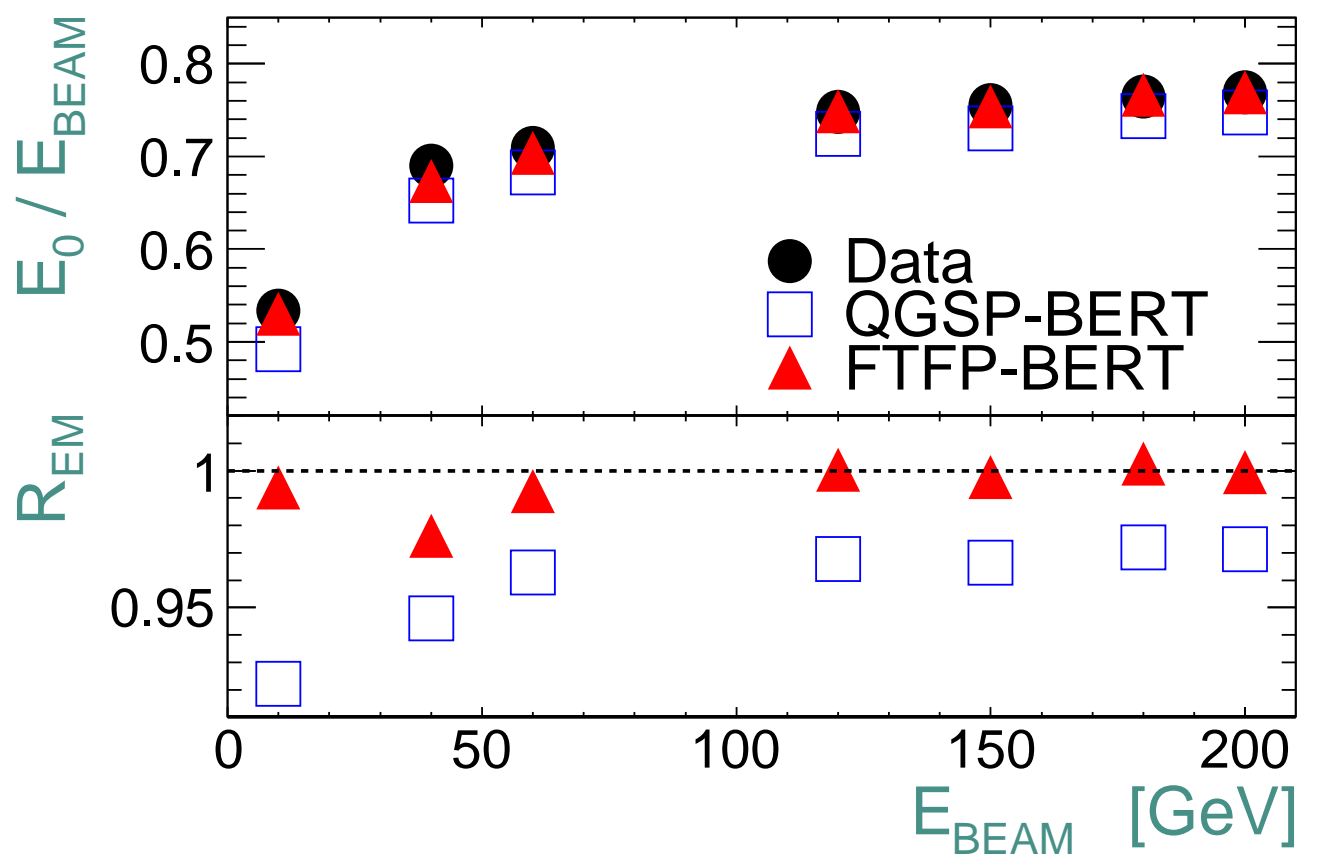

- Non-linear response reflects the non-compensating nature of the FCal

- FTFP-BERT describes the experimental response well

- QGSP-BERT underestimates the response by 3-8 \% 
Charged pions in the forward region

\section{Energy response at LC-scale}
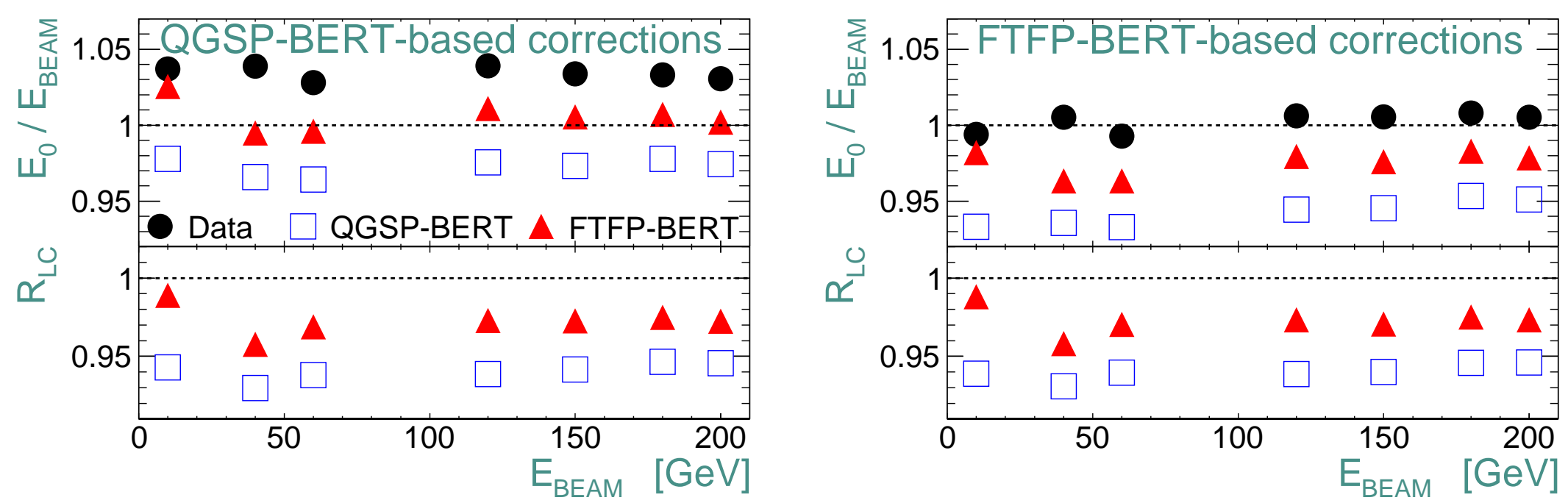

- Experimental response is best reconstructed with FTFP-BERT-based LC-corrections

- Application of native LC-weights and corrections to the corresponding simulations yields an underestimate of the response by $2-4 \%$

- A good agreement between the experimental response and response predicted by FTFP-BERT existing at EM-scale becomes worse at LC-scale (up to $3 \%$ )

- For QGSP-BERT a disagreement observed at EM-scale becomes larger at LC-scale 
Charged pions in the forward region

\section{Energy response (cont'd)}

\section{Difference in the response for QGSP-BERT and FTFP-BERT physics lists}

- $\sim 3 \%$ at EM-scale

- $\sim 0 \%$ after application of the native LC-weights and corrections

- $\sim 5-7 \%$ after application of the non-native LC-weights and corrections

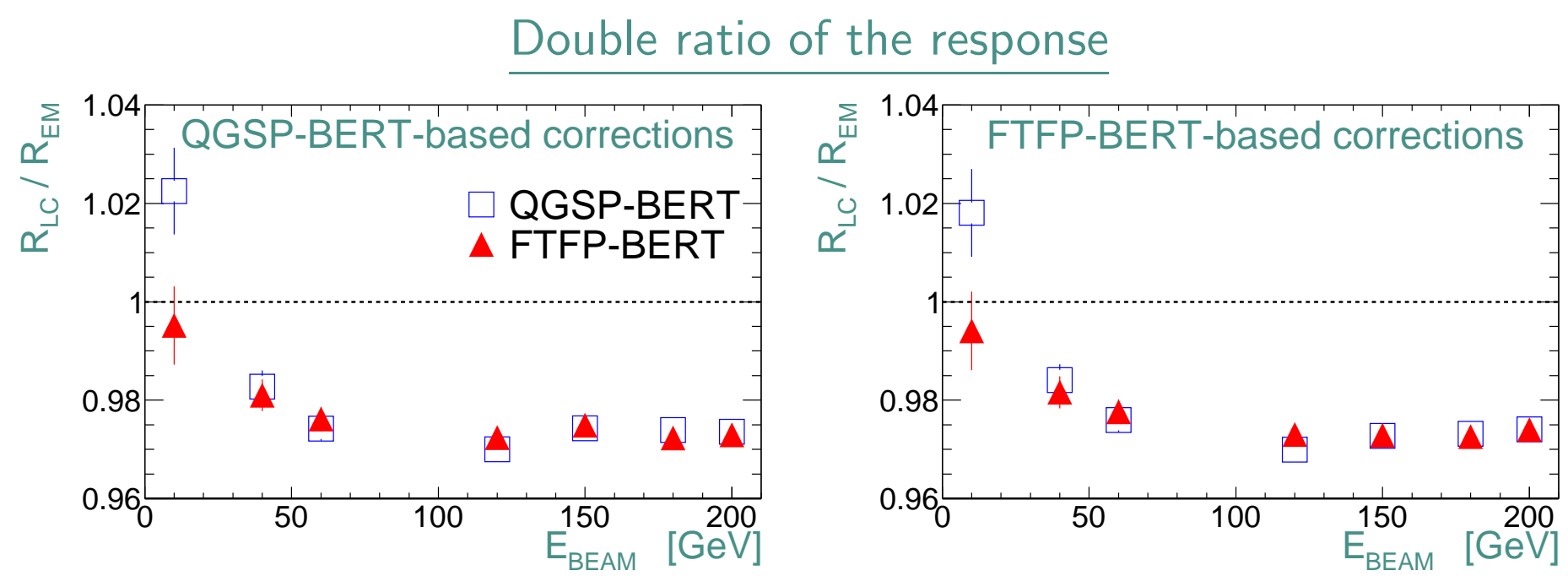

- Double ratio of the energy response differs from unity up to $3 \%$

- Results obtained for both physics lists are similar 
Charged pions in the forward region

\section{Energy resolution}

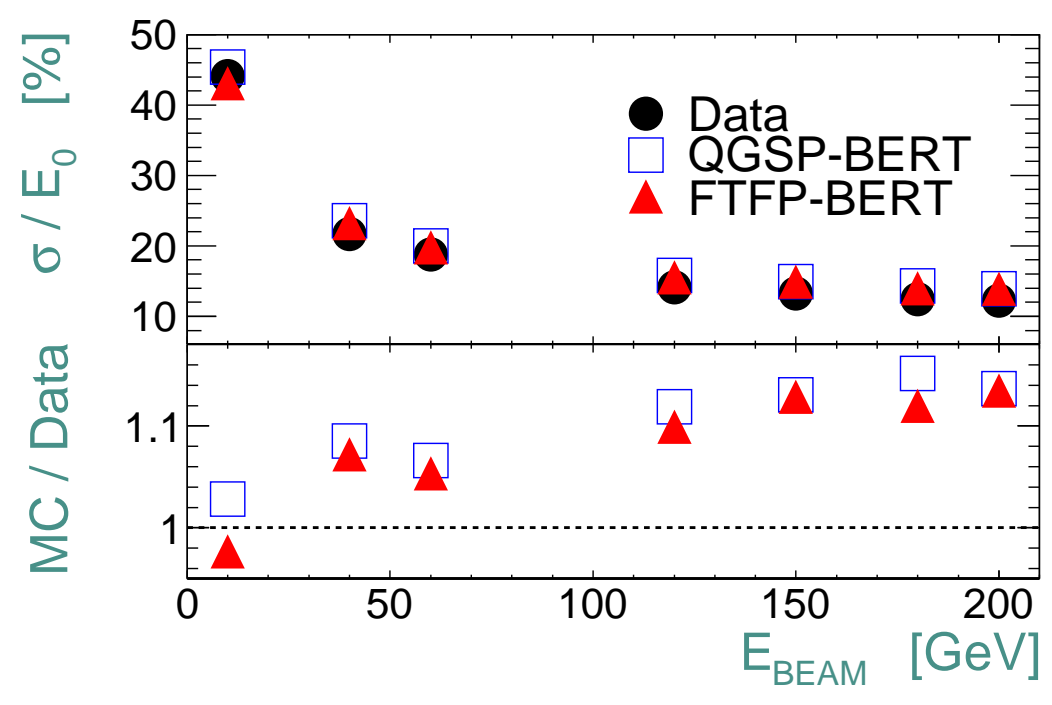

\section{Successive application of LC-steps}

- Weighting step improves the energy resolution at higher beam energies

- Out-of-cluster corrections improve the energy resolution at lower beam energies $40 \mathrm{GeV}$ )

\section{Resolution at EM-scale}

- Two physics lists give similar results (for $E_{\mathrm{BEAM}} \geq$

- They predict worse energy resolution at EM-scale than experimental values by $5-15 \%$ there

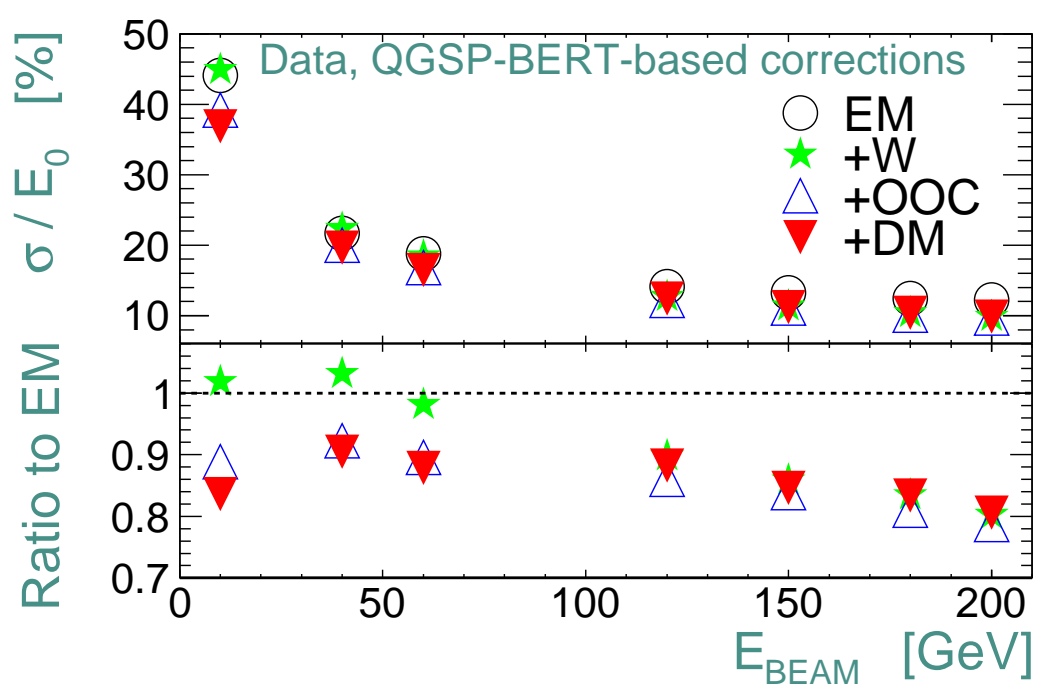


Charged pions in the forward region

\section{Energy resolution at LC-scale}
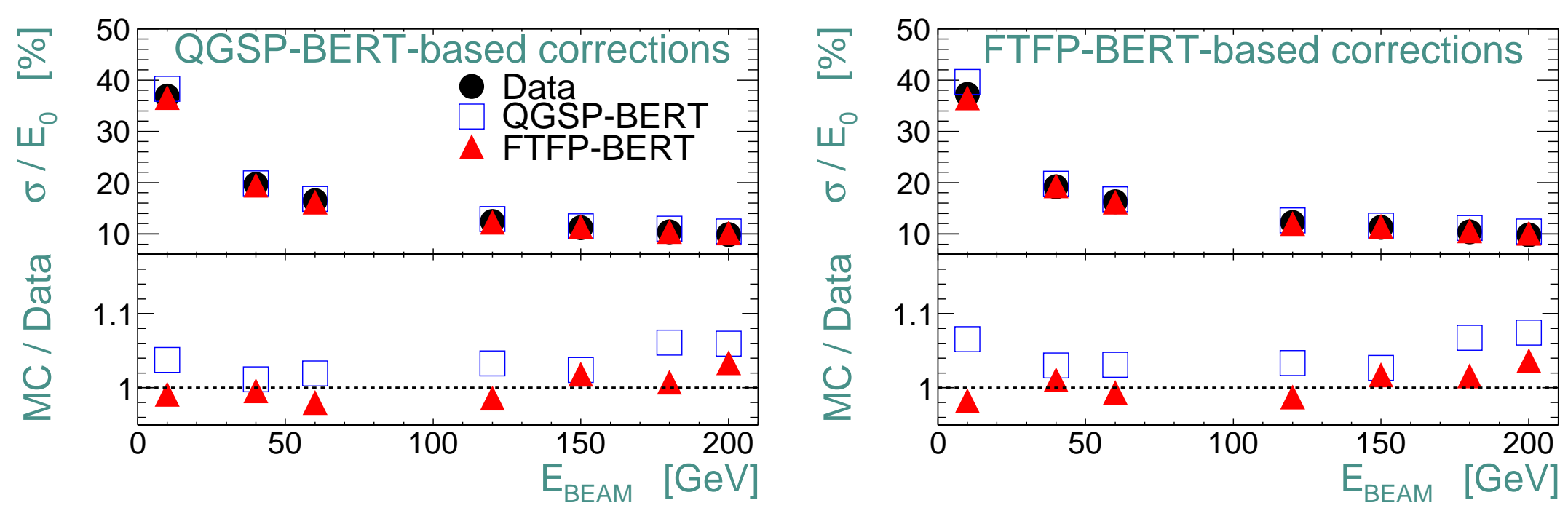

- After application of the local hadronic calibration the resolution in general improves by $10-25 \%$ for both data and simulations

- The local hadronic calibration significantly improves the agreement between experimental and predicted values of the resolution, especially for the FTFP-BERT physics list 


\section{Vertical scan with charged pions}

- Vertical scan over the entire calorimeter system, corresponding to the pseudorapidity scan in the ATLAS coordinate system: $2.5<\eta<3.8$

- Covers:

- EMEC and HEC: $\eta<3.2$

- FCal: $\eta>3.1$

- crack between the end-cap calorimeters and the FCal around $\eta \sim 3.2$ (non-projective)

- support structure positioned in front of the FCal at $\eta \simeq 3.3$ (projective in ATLAS)

- Beam of $200 \mathrm{GeV}$ charged pions

- MC simulations of the vertical with the two physics lists: QGSP-BERT and FTFP-BERT

- Position reconstruction:

beam $Y$-coordinate in the beam test system is converted to the pseudorapidity $\eta_{\mathrm{BEAM}}$ in the ATLAS coordinate system by using the correlations between $(X, Y)$ - and $(\eta, \varphi)$-coordinates of reconstructed clusters

- Energy reconstruction:

- sum of energies of all clusters

- parameter $E_{0}$ as the mean of energy distributions

- parameter $\sigma$ as the RMS of energy distributions

- Analysed variables (as functions of $\eta_{\mathrm{BEAM}}$ ):

- cluster moments: energy density and shower depth

- pion energy response $E_{0} / E_{\mathrm{BEAM}}$

- pion energy resolution $\sigma / E_{0}$

- LC-scale: QGSP-BERT-based corrections 


\section{Cluster moments}

\section{Energy density}

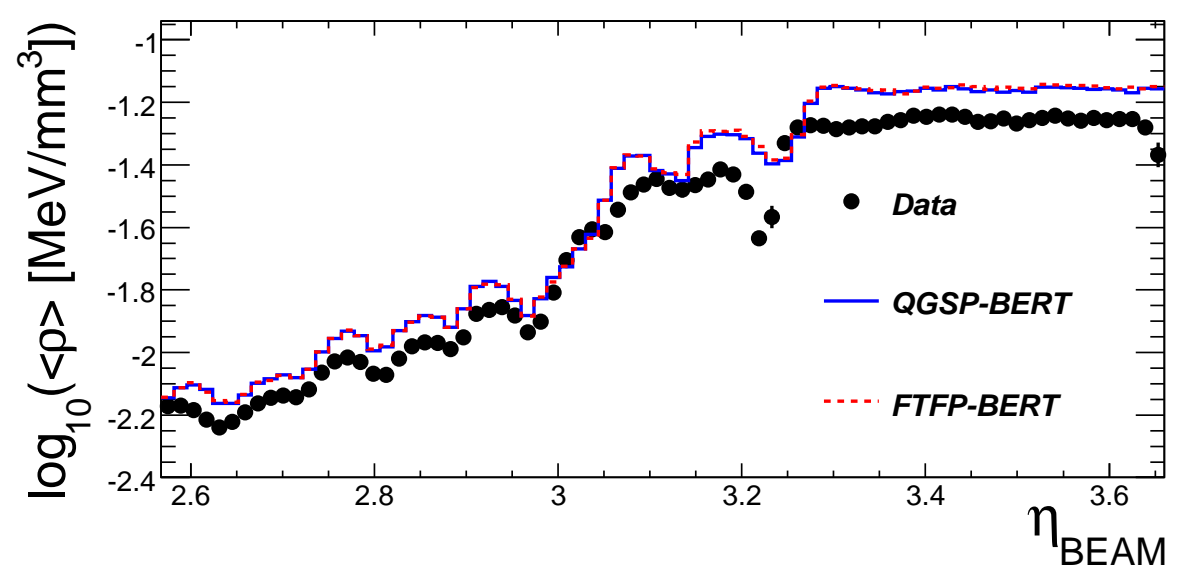

- Pseudorapidity dependence of the energy density predicted by both simulations follows the experimental behaviour over the studied $\eta$-range

- But the values from simulations are shifted to higher densities relative to the data, as both physics lists predict more compact hadronic showers than observed experimentally

\section{Shower depth}

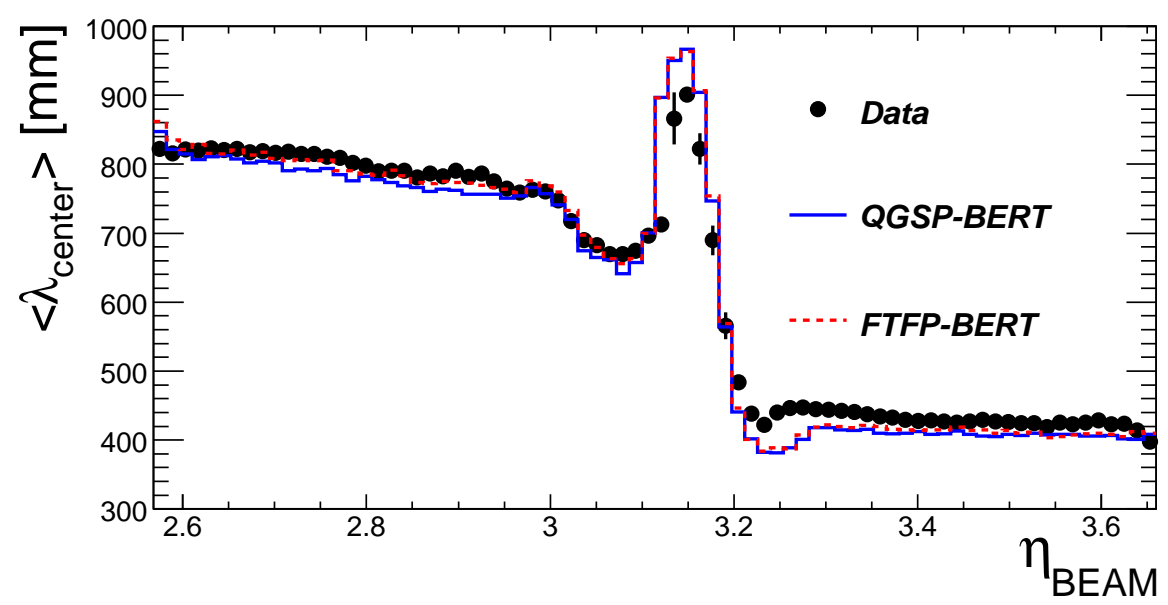

- Pseudorapidity dependence of the shower depth is rather constant across the end-cap and forward regions but varies significantly in the crack region

- Agreement between simulations and experimental data is rather good over the studied $\eta$-range

\section{Agreement between the two simulations is very good}


Vertical scan with pions

\section{Energy response at EM-scale}

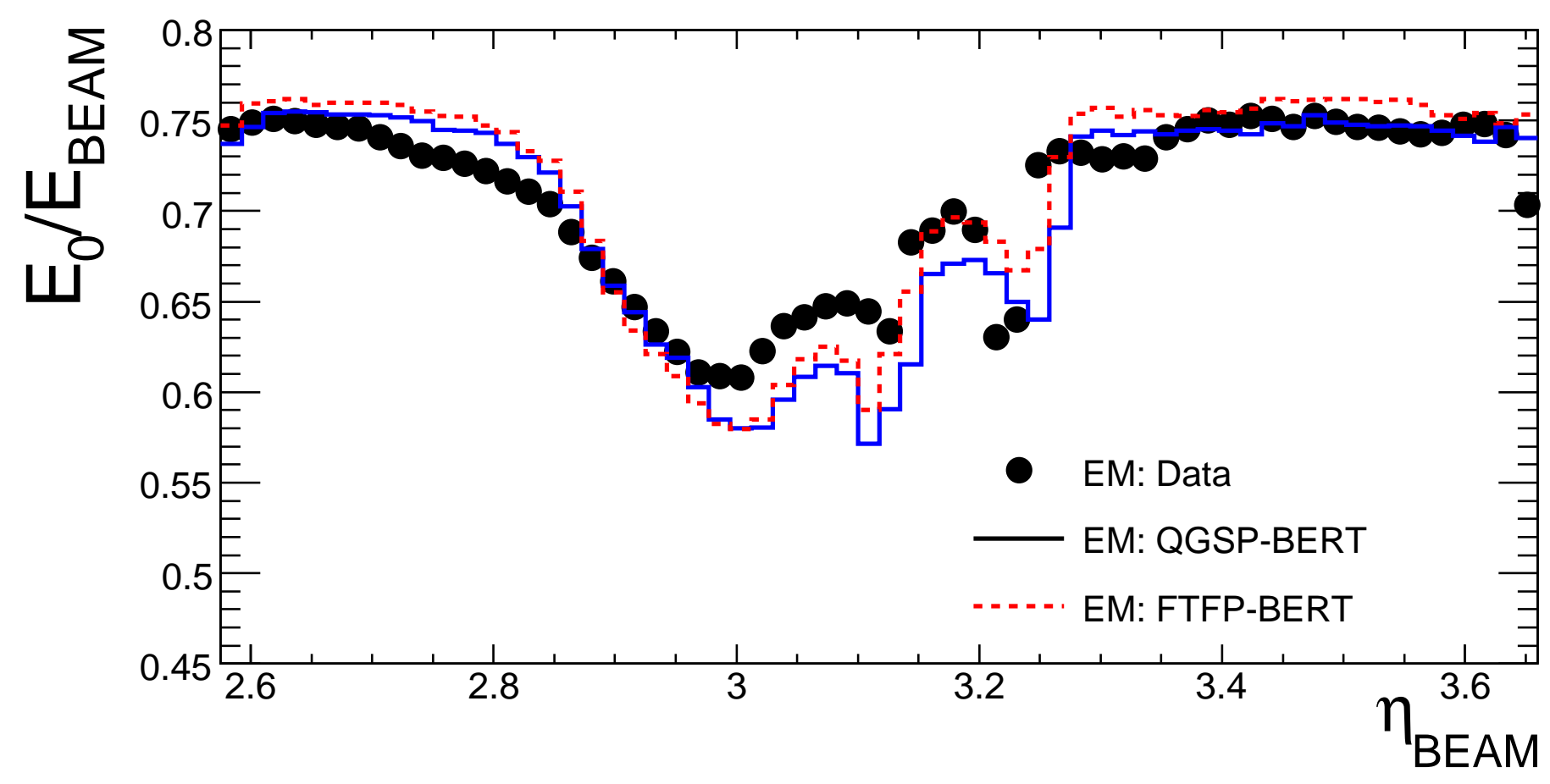

- Agreement between data and simulations is reasonably good outside the crack region and becomes worse in the crack region

- Smooth drop of the response with the beam pseudorapidity in the end-cap region is due to the decrease of energy of clusters reconstructed closer to the crack between calorimeters 
Vertical scan with pions

\section{Energy response at LC-scale}

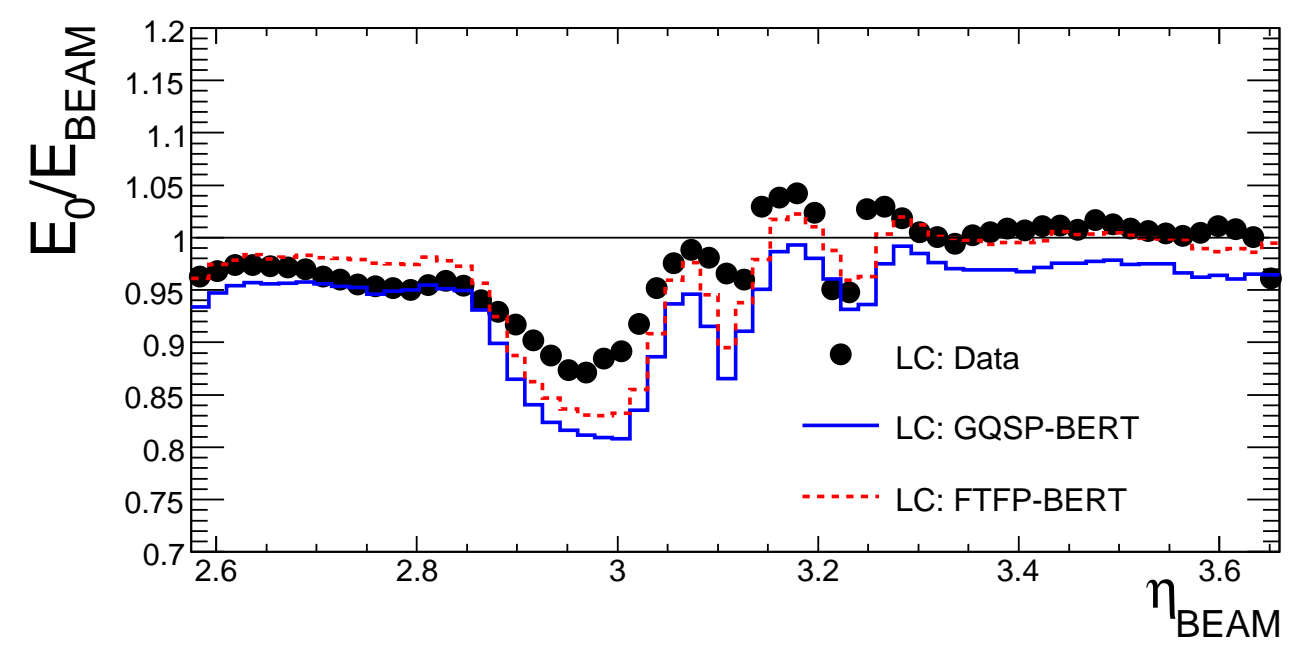

- The energy response becomes at LC-scale close to one, as expected.

- The agreement between experimental data and simulations outside the crack region is moderate. In the crack region simulations follow the data.

- FTFP-BERT describes the experimental response at LC-scale for the full vertical scan better than QGSP-BERT.

- With respect to EM-scale the response dip in the crack region becomes smaller.

- Nevertheless the average energy response here is lower than $E_{\mathrm{BEAM}}$ by $10-15 \%$ for the data:

- low reconstruction inefficiency

- cluster misclassification 


\section{Vertical scan with pions}

\section{Energy resolution}
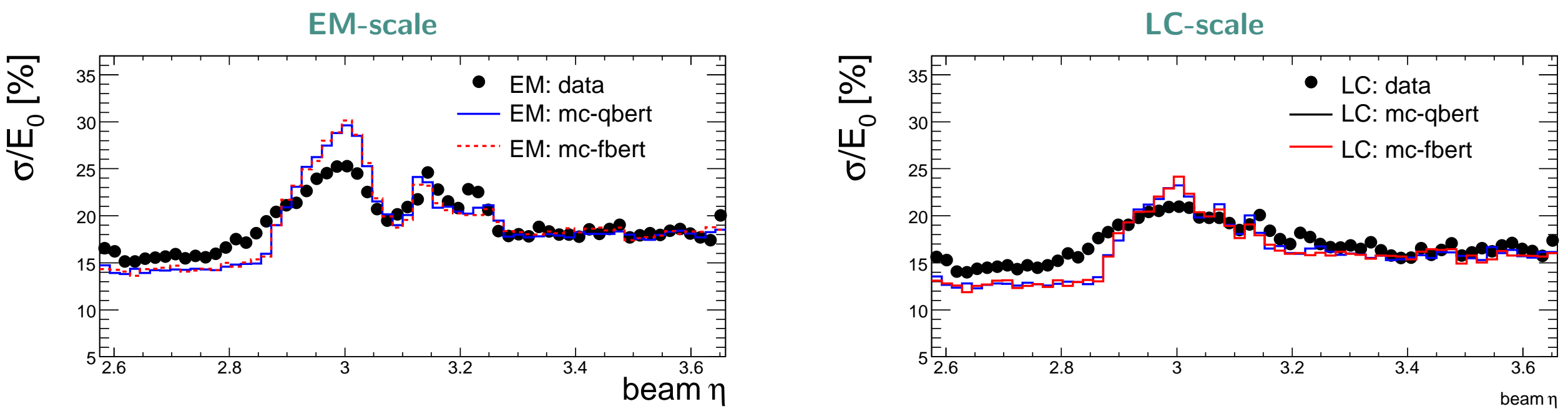

- Significant degradation of the resolution at EM-scale is observed in the crack region $(\eta \sim 3.2)$ and in the region around $\eta \sim 3.0$

- In the latter case this is related to the changes of the cluster size near the boundary of the end-cap calorimeters, where the drop in response is observed as well

- There are also differences between data and MC predictions in this pseudorapidity region, which can be explained by more compact hadronic showers in simulations

- At other values of the pseudorapidity the agreement between data and simulations is rather good

- At LC-scale the resolution is improved for the full scan range, especially in the regions around $\eta \sim 3.0$ and $\eta \sim 3.2$

- Agreement between data and MC simulations also becomes better at LC-scale 


\section{Conclusions}

The local hadronic calibration is an advanced method for reconstruction of energy deposited in the ATLAS calorimeter system. It uses modular corrections applied to cluster energies reconstructed at EM-scale.

This method is validated with data obtained during beam tests of modules of ATLAS endcap and forward calorimeters, corresponding to the pseudorapidity range $2.5<|\eta|<4$.0. Validation is performed using detailed GEANT4 based simulations of combined beam tests.

The local hadronic calibration allows to reconstruct the initial energy of charged pions in the end-cap region with uncertainty below $1.5 \%$. In the forward region the uncertainty of the response is about $3 \%$. In the complicated transition zone between the calorimeters (including a crack at $\eta \sim 3.2$ ) the response is underestimated by $10-15 \%$.

One of the important features of the local hadronic calibration is a significant improvement of the energy resolution in all studied calorimeter regions. 